\title{
Symbols, metaphors, analogues: seeding, modelling and achieving sustainable design
}

\author{
R. J. Koester \\ Ball State University, USA
}

\begin{abstract}
This paper describes the role of abstraction in providing informational structure to the complex tasks of the design-for-sustainability process and the use of that abstraction in facilitating the participation of the many players needed to assure the successful execution of projects. The distinction is made between "seeding" and "modelling" as parts of an iterative function in service to design-for-sustainability. Distinctions are drawn between the trappings of the labelling and symbology associated with systems used to index sustainable performance (such as the US Green Building Council LEED Rating System), the role of both computer-based and hands-on simulation/emulation tools used to quantify sustainable performance, and the inspirational evidence to be found in nature as exemplary embodiments of sustainable performance - all of which can contribute support to the integrative process needed to assure the effective pursuit of design-for-sustainability. Specifically, discussion is provided of the distinctions between symbol, metaphor, and analogue. In addition, critique is made of Ecological Design and Open Building Design as used to promote sustainable design. Only by clarification of the respective distinctions and with a thorough understanding of the strengths and weaknesses of each of these, as a tool, can we be prepared as designers to seed and model - and thereby effectively achieve - a sustainable architecture.
\end{abstract}

Keywords: symbol, metaphor, analogue, modelling, design process, green design, ecological design, open building, sustainable design.

\section{Introduction}

In the face of the pervasive social concern for environmental, social and economic sustainability and the need to find ways to integrate this content into 
the design, delivery and operation of sustainable facilities, numerous ideas are put forward; terms of definition are invoked and references are made to describe the complexity of this challenge. Nonetheless, confusion readily occurs. Terms such as green design, sustainable design, and ecological design are used interchangeably. Well-intentioned rating methods such as LEED, Green Globes, and BREEAM equally can serve to cloud the conversation.

As designers, our first response to the confusion is to find ways to gain control over the overwhelming nature of this information. The use of design guidelines, lists of issues, performance diagrams and singular terminology all reflect an effort to gain that control. This paper examines this landscape to propose a specific, rather simplified means by which conceptual abstractions can serve to clarify and give informational structure to this otherwise confounding new field. More specifically, the interest is to preserve the useful operation, intent and understandings of the traditional design activity so as to leverage the proposed abstraction as a service to the looming goal of design-for-sustainability.

\subsection{Abstraction as Informational Structure}

The most common operational tool used by architects and allied design professionals to give structure to complexity is the infamous "back of the envelope" diagram. Such a diagram often illustrates proposed performance and employs what are best described as "smart" arrows. The illustration typically patterns the flow of environmental forces in desirable ways but is not necessarily derivative of an actual mapping of the true effect of the built-form-influence on such environmental force flow. In addition to the diagram of course, design professionals frequently use the "back of the envelope" label which seeks to capture core ideas not only for talking with clients and consultants, but also to 'spirit' the project by painting a mental image to which participants can aspire.

In either case, whether using a diagram or label-or even a sentence or paragraph imagining of the design intent - the purpose is to give structure to information. The challenge to design-for-sustainability is to systematize the conventions of abstraction so as to better support the design process; and especially to assure the clarity of roles, and active participation, by all players.

\subsection{Sustainable design in all its complexity}

Sustainability as a topic and the design of sustainable facilities as a task allude to a complexity that exceeds the fundamental understandings of building construction; this is true as well in landscape design. Quite separate from the complexities of material choice, assembly and operation, which comprise the standard accountability in facility or landscape design, the invocation of the sustainability mantra necessitates even more careful monitoring of the metricswith specific attention to the expected behaviour of day-to-day, life-long operational performance. This necessary obsession with tracking flows and balances so as to assure sustainability, frequently involves the use of computer software modelling and/or hands-on simulation techniques by which the predictability of operational behaviour is given some sense of certainty. 
Such complexification of the process demands evermore clarity in the informational abstraction and organizational structure used to affect the process of design and the delivery of a fully functioning final project. This is exacerbated even further when considering the need to move from the more sequenced (asynchronous) contribution by the players to the more simultaneous (synchronous) interaction of the many allied professionals.

\subsection{Early participation by all}

The sequence of conventional design service delivery which aggregates activity in phases from site selection and analysis, to programming, to schematic design, to design development, to construction documentation, to bid supervision and even post-occupancy evaluation necessitates a new look when faced with the complexities of sustainability. A more appropriate approach recognizes that all participants should be at the table from Day 1 so that the overlapping influence of concerns on the wholeness of the project can be examined, debated, and cyclically edited in an expansionist/contractionist charette process. This needed participation by all players throughout the process emphasizes the utility of simplified abstraction in giving structure to the communication of interaction.

\section{Narrative}

In many ways the challenge we face in seeking to design sustainable facilities is the challenge of picking a starting point in the process. The conventions of design practice and the staged sequencing of design development as mentioned above typically point toward the use of checklists to enrich the standard model of design service delivery while not changing it fundamentally. But the integrated nature of sustainable performance argues in favour of changing the process; the sections which follow present the means by which such change can occur.

\subsection{Seeding is not modelling}

The starting point for any design is a 'seeding' exercise; that is to say one must have some notion of organizational purpose, diagrammatic arrangement, material selection, and/or mandate from the client that sets in place a first definition of the fixed and the variable in the decision making tree. 'Seeding', however, is not the same as 'modelling' and it is important to get at the distinction between the two. They are linked, even closely aligned; but, they are radically different. For purposes of this paper, 'seeding' is defined as having to do with ordering ideas and 'modelling' is defined as having to do with operational behaviour.

\subsubsection{Seeding}

Many stories are available regarding the beginning point of design. Architects have been known to start with a building (as a type) that they have designed before and to look toward modifying its plans and sections as a new fit in a new circumstance (client, context, site, etc.). This approach reflects a kind of artificial structuring of problem complexity in that the imposition of the seeding 
idea although seeming somewhat arbitrary intentionally begins as a "bad" fit (in detailed terms) so as to make it possible to enter the complexity of the problem space at a high level of organizational control - thereby leading to the meaningful genesis of a subsequent "good" (more sophisticated) design fit. The blessing of such approach is that one starts quickly; the accompanying curse is that one may lose the histories of rationalization embedded in the seeding idea. Nonetheless, this is a tremendously useful technique to kick-start a new project.

Many stories can be told. In tracing the work of any great architect we can see in subsequent work the seeds of design ideas embedded from prior projects. These show up often in literal terms - in the plan and/or section footprints of the buildings, the choice of materials, the proportionalities of space, the geometries of alignment and/or the placements of the buildings on the land. Often these reflect a continually evolving basis by which specific responses to environmental forces are made.

\subsubsection{Modelling}

In contrast, the modelling of problem solutions and ramifications of design decision-making can be carried out using accepted "tools of the trade" such as checklists, design guidelines, code-based mandates, strictures for established best practices, and/or computer software. In these cases, the modelling is actually a projection of expected operational performance based on the known behaviour of the pre-existing proven design. A consultant, who applies this approach as an energy-system modeller, for example, will frequently start with a go-to model of a known building (by type) and simply 'tweak' it to approximate a proposed design; informing and challenging the conversation about the engineering of force flows and the management of systems behaviour.

The point to be made involves the distinction between 'seeding' as a real-time action meant to precipitate real-time behaviour of participants in the design process and 'modelling' as a virtual-time emulation (or mimicking) of the expected real-time behaviour of the building. It is important also to discuss how the design process can be driven by a visionary inspiration.

\subsection{Inspirational evidence in nature}

Nature herself is one of the best teachers regarding the visualization of design process and the interactive characteristic of limits and expression. We could discuss the inspirational aspect of nature by connecting the potentiation of DNA to expressions of cellular (read built) growth in response to environmental constraint. We could expand this to include a Darwinian observation regarding the development of species (read building type) and the differentiation of each of these relative to their respective operational islands (read biomes, climates, and building sites). More generally though, the invocation of nature is helpful in talking about operational principles.

\subsubsection{Location, form, metabolism}

Nature teaches that animals and plants function in response to environmental force by modifying location, form and/or metabolism. Animals, for example, 
migrate from point A to point B to escape the less favourable, or to enjoin the more favourable conditions; both animals and plants differentiate form to expand or contract exposure to the elements; and animals activate metabolic function to compensate for environmental stress. These rather straightforward teachings are found in the migrations of birds or butterflies from winter to summer seasons; the form (and size) of Elephant ears used to promote thermal exchange; the on-the-spot shivering by animals as a metabolic activity in direct response to the environmental constraint.

\subsubsection{Form, operation, appearance}

These factual aspects of physiologic fit of a species to environment can be contrasted also against the more poetic inspirational evidence in nature. It is not uncommon for designers to invoke themes of form, operation, and/or appearance in that regard. The bird-wing quality of the Milwaukee Art Museum by Calatrava (Aldersley-Williams [1]), the differentiation of the skin of the Phoenix Public Library by Bruder (Wigginton and Harris [2]), or the physical form of the Fish Dance Restaurant by Gehry (Aldersley-Williams [3]), exemplify this point.

\section{Trappings}

The conventions of practice as referenced in the introduction yield a kind of trap for the designer; when burdened by the labels and symbols invoked.

\subsection{Labelling}

One example of labelling, established as a formal evaluative system, is the US Green Building Council Leadership in Energy and Environmental Design (LEED) scoring technique. With this method, one can obtain designation for a building as operating at levels of performance meriting the ratings of certified, silver, gold, or platinum. Proponents of the system even argue in favour of "exceeding LEED" by achieving living building performance. The simplified scoring system which involves a substantial interactive review process reflects a disassociation of actual performance and operational behaviour at the level of content and supplants that measured performance with the simplified terminology of the label itself. In fact, the most recent literature is reporting that some of the LEED certified buildings are not performing as intended (Schendler [4]); in part because some have achieved their scores while paying minimal attention to energy flows - an important element of sustainability.

\subsection{Symbology}

In contrast to the scoring method and the trappings of the certification labels, there is a latent symbology embedded in popular design terms such as green, ecological, sustainable and even regenerative design. These are used often in overlapping and interchangeable ways and lack clear agreed-upon definitions. Is a green building a sustainable building or is a sustainable building green? Can a living machine alone make a building green? Is a building green only when 
measured in terms of materials or is it green because of its energy behaviour? Is a sustainable building only sustainable because of the sourcing of material or because of its operational performance? The confusions aggregate quickly.

The point to be made is that we must guard against the trappings of language and we must be careful not to invoke symbology as a substitute for content when faced with the tasks of design-for-sustainability. One of the most tried and true examples of symbolic invocation is the use of the sun path diagram on a building plan or section. The minute one puts this on the sheet, the building is 'labelled' a solar building and the presumption is that it will perform at some level of accepted best practice. What the labelling does not do is explain in any detail what the 8760 hour track of performance will be during a typical meteorological year, nor does it get at the interaction of the various systems or design strategies used to assure some level of expected operational performance.

\section{Tools}

There is a wide range of computer software on the market by which one can simulate the behaviour of intended building performance. This includes energy, lighting, acoustics, finances and the life-cycle evaluation of material impacts.

\subsection{Thermal}

The most robust energy modelling tool in the United States is DOE-2 which requires a complex description of building energy components; it uses sophisticated thermal network modelling to mimic the trade-off of energy gains and losses in the capacitance of a building's spaces and materials over the course of days, weeks, and months - as influenced by the dynamic occupant and climate loads. Other tools, such as Energy Plus, Energy-10, Energy Scheming (a more qualitative inferencing tool), and Eco-Tec all utilize similar network modelling of energy flows as thermal capacitance trade-offs. Nonetheless, even the most sophisticated application of these modelling tools does not yield absolute assurance of final performance. The tools simply put a design idea "in the ballpark"; more importantly they give reasonable assurance that the system choices made have a mutual, internal integrity of performance as they interact.

\subsection{Luminous}

The most prominent tool in lighting simulation is Radiance which is used to create pictorial imaging of rendered computer models with a back-up technical accounting of performance metrics. The software offers effective visualizations of expected final appearance of light behaviour in a space based on known reflectivity and co-efficiencies of absorption for materials selected as room surface finishes. Factored into such models are fairly accurate predictions regarding the influence or impact of hard light from the sun and the inter-reflective light from the sky-vault. The sophistication of these rendering capabilities, however, falls short of the need for quick iterative feedback during the process of design. As a result, many lighting designers use more immediate 
modelling techniques such as Heliodon and/or Sky Chamber Daylight emulators which enable the participants to work in real time to make quick decisions and test options 'on the fly'. Most importantly, these modelling tools are useful in feeding and/or otherwise supporting conversation among the allied professionals contributing to the work, since all of them can be at the table at the same time.

\subsection{Acoustic}

A popular software tool for acoustical evaluation is CATT-Acoustic, which calculates the reverberation effect and inter-reflective accumulation of wave phenomena in a physical space based on material choices, space geometry and contained air volume. The tool is quite helpful in emulating the acoustical quality and can lead to an understanding of the expected technical regimes for reverberation and the noise deadening effects of materials and space geometry. Nonetheless, in the end, acoustical spaces are only able to be fine-tuned (perfected) using more empirical trial-and-error methodologies in the field; "sparking" a point signal, measuring actual reverberation time and profiling the frequency response of a space can only be made on location in real-time.

\subsection{Financial}

Numerous tools are available for doing life-cycle cost (LCC) analysis and/or day-to-day return on investment (ROI) profiling using assumptions about capital costs, discount rates, longevity of the project, annual operating expenses, and other variables of influence. These financial models can be used to track the trade-off of higher front end costs against lower operational costs yielding a cost-benefit over time. Interestingly, these modelling tools enable the capture of the interactive nature of system design; buildings that score LEED Silver often cost less to effect than those meeting Certified rating. The internal trade-offs and multiplying effects of the conservation efforts accumulate with the greater level of sophistication indicated by the higher performance rating.

\subsection{Life Cycle}

In contrast to costs, tools are available for determining a Life Cycle Assessment (LCA). These measure the net environmental impact of materials as tracked from the point of sourcing through manufacture to delivery and operation. The more notable software tool on the market that engages this kind of profiling is that of the Athena Software Group. Life Cycle Assessment also looks at operational use in place and tries to establish the benchmarks for replacement or repair needed during the ongoing maintenance and operation of a facility. Both of these involve a sophistication that exceeds conventional architectural service.

\section{Discussion: a wilful simplification}

In the light of the discussion above, there is need for clarity of conceptual structure. The three terms discussed below are offered as that device. They 
comprise a kind of graded invocation of understandings that distinguish the trappings, inspirations, and metrics of design ideation-especially as these involve all contributing parties working in service to design-for-sustainability.

\subsection{Symbol}

For sake of definition, the symbol is seen as iconographic; a signal of intent, a marker of content, but is not itself considered operational. As described above, the symbol "stands for" operational performance as a measure of sustainability. The LEED rating system, the sun-path diagram, or even the labelling of a plan and/or building cross-section with so-called smart arrows, tend more to be "calls for" intended performance rather than measurable predictions. Such symbols need to be qualified for what they are to distinguish their more superficial level of information structuring from those which are more penetrating.

\subsection{Metaphor}

For the sake of definition, the metaphor is seen as an allusion and is used to establish a spirit for the project. A building can be imagined to be "as a bird on a nest"; or seen to be structured "as a spider's web", or could have experiential qualities "as in the space of a cave". Metaphors allude to a qualitative feel as well as an organizational inference, but do not talk to specific operational practice or expected (let alone, measured) performance.

\subsection{Analogue}

By contrast, the term analogue is used to talk about the operational aspects of models - especially those in nature - that can be used to inform design. Analogues are those physical performances that can be mimicked in built form. The chimney-effect ventilation of a tall building as analogous to the natural stack-effect ventilation of the termite mound is a profound example.

\section{Critique}

The proposal herein is to use the three terms above to describe, interpret, and/or otherwise employ the widely-available labels, diagrams and tools of design-for-sustainability. Moreover, the goal is to leverage other bodies of architectural work and schools of thought whose origins and/or histories do, or do not, have an immediate connection. To emphasize this point, the following two critiques are made of Ecological Design and Open Building, respectively.

\subsection{Ecological design}

Much of the concern for sustainability in architecture and allied design fields operates under the rubric of ecological design. A simple web search yields all manner of definitions and/or interpretations of the term. There are numerous practicing professional groups and individuals that weave this into the titling of 
their company; design-build and professional schools of architecture throughout the world invoke the term as part of their curricular descriptions, if not degree titles; and various manufacturers invoke the term to describe their products. Organizations, institutes and centers use this term as well; one of the most notable is The Ecological Design Institute (Van der Ryn [5]) which offers namesake guidelines: (1) Solutions grow from place (2) Make nature visible, (3) Design with nature, (4) Ecological accounting informs design, and (5) Everyone is a designer. Another checklist example comes from Malcolm Wells, the so-called underground architect whose work predated much of the contemporary concern for environmental, economic and social equity formalized as the sustainability interest; Wells compiled an intriguing matrix by which designers could score their projects - whether buildings or landscapes. The list provides a simple polarity of qualitative measures regarding how a design "contributes" to the creation of pure air or destroys it, "creates" pure water or destroys it, etc. (SBSE [6]). A forest landscape, of course, merits a perfect score.

\subsection{Open building}

Of the many schools of thought that have bearing on the process of facility delivery as well as use, Open Building is perhaps one of the more internationally recognized in the literature. Open Building aggregates design decisions in time and space on five levels - urban structure, urban tissue, base building, fit-out, and furnishings. And between each of these, defines the territorial units of town, neighbourhood, functional areas (or departments), and rooms. The concepts grow from the early writings of Habraken [7] regarding supports and infill as a means of conceiving elements of a building which have differing physical lives. Open Building aligns itself with the idea that since base buildings and urban infrastructure have the longest lives of the systems, they can be designed and evaluated using a 100 year life-cycle.

The literature on Open Building is well developed, but the integration of sustainability factors is not fully developed or well refined (Koester et al. [8, 9]). The categorizations of symbol, metaphor and analogue can help Open Building designers address more effectively the issues of environmental fit at each of the levels, and appropriately import the resource materials needed to support the nested levels of decision-making.

\section{Conclusion}

In the face of the sustainability concerns, the challenge is to find ways to bring all players to the table using a language of categorization by which discussion can be anchored. The proposal in this paper is to use the simple hierarchy of symbol, metaphor, and analogue as a way to stimulate kinds of discussion, categorize available resources, identify design process activities, and inventory design documentation. The simplicity of the terms and the clarity of their definition will promote dialog and establish a hierarchy of appreciation for the complexity of the task of achieving sustainable design. 
184 Eco-Architecture: Harmonisation between Architecture and Nature

\section{References}

[1] Aldersey-Williams, H. Zoomoorphic: a new animal architecture. HarperCollins: London, pp. 50-51, 2003.

[2] Wigginton, $\mathrm{M}$ and Harris, J., Intelligent skins. Elsevier, Amsterdam, pp.99-102, 2002

[3] Aldersey-Williams, H. Zoomoorphic: a new animal architecture. HarperCollins: London, pp. 47, 2003.

[4] Schendler, A and Udall, R., LEED is broken; let's fix it., 2005 http://www.grist.org/comments/soapbox/2005/10/26/leed/index1.html.

[5] Van DerRyn, Sim., The ecological design institute., 2005 http://www.ecodesign.org/.

[6] SBSE, A regeneration-based checklist for design and construction., 2005 http://www.sbse.org/resources/index.htm.

[7] Habraken, N.J. The structure of the ordinary: form and control in the built environment. MIT Press: Cambridge, pp. 1-100, 9999, 1998.

[8] Koester, R., Open building and community harvest: new definitions of what can comprise a base building. Proceedings of the 2005 World Sustainable Building Conference, Tokyo, pp. 2976-2982, 2005.

[9] Koester, R., Dettbarn, D., and Riegle, E., Tectonic constraint: harvesting material(s) -- and building(s) -- to seed sustainable architecture. Proceedings of the $21^{\text {st }}$ International Conference Passive and Low Energy Architecture (PLEA), Eindhoven, pp. 571-576, 2004. 